\title{
BMJ Open Broad Spectrum project: factors determining the quality of antibiotic use in primary care: an observational study protocol from Italy
}

\author{
Peter Konstantin Kurotschka (D) , ${ }^{1,2}$ Alice Serafini, ${ }^{3}$ Marco Massari, ${ }^{4}$ \\ Roberto Da Cas, ${ }_{4}^{4}$ Adolfo Figueiras, ${ }^{5}$ Viviana Forte, ${ }^{6}$ Maria Francesca Moro, ${ }^{7}$ \\ Matteo Massidda, ${ }^{2}$ Federico Contu, ${ }^{2}$ Luigi Minerba, ${ }^{1}$ Maurizio Marcias, ${ }^{8}$ \\ Marco Nardelli, ${ }^{9}$ Alessandra Perra, ${ }^{1}$ Mauro Giovanni Carta (D) , ${ }^{1}$ \\ Stefania Spila Alegiani ${ }^{4}$
}

To cite: Kurotschka PK, Serafini A, Massari M, et al. Broad Spectrum project: factors determining the quality of antibiotic use in primary care: an observational study protocol from Italy. BMJ Open 2020;10:e038843. doi:10.1136/ bmjopen-2020-038843

- Prepublication history and additional material for this paper are available online. To view these files, please visit the journal online (http://dx.doi. org/10.1136/bmjopen-2020038843).

PKK and $A S$ are joint first authors.

MGC and SSA are joint senior authors.

Received 26 March 2020

Revised 08 May 2020

Accepted 12 May 2020

Check for updates

(C) Author(s) (or their employer(s)) 2020. Re-use permitted under CC BY-NC. No commercial re-use. See rights and permissions. Published by BMJ.

For numbered affiliations see end of article.

Correspondence to Dr Peter Konstantin Kurotschka; kurotschka@hotmail.com

\section{ABSTRACT}

Introduction The overuse of antibiotics is causing worldwide spread of antimicrobial resistance (AMR). Compared with other countries, Italy has both high antibiotic consumption rates and high rates of AMR. Due to the fact that around $90 \%$ of antibiotics are prescribed by general practitioners (GPs), this study aims to measure the impact of knowledge, attitudes and sociodemographic and workplace-related factors on the quality of antibiotic prescriptions filled by GPs in the Italian Region of Sardinia. Methods and analysis Knowledge, attitude, sociodemographic and workplace-related factors deemed to influence physicians prescribing behaviour will be evaluated in a cross-sectional study conducted among all GPs of the Italian Region of Sardinia $(n=1200)$. A knowledge and attitudes questionnaire (Knowledge and Attitudes on Antibiotics and Resistance - Italian version: ITA-KAAR) accompanied by a sociodemographic form will be linked to drug prescription data reimbursed by the National Health System. European Surveillance of Antibiotic Consumption quality indicators for outpatient antibiotic use will be calculated from drug prescription records. Every GP will be deemed to have demonstrated an adequate quality of prescriptions of antibiotics if half of the indicator score plus one is better than the median of the region. A multivariate Poisson regression model with robust variance estimation will be used to evaluate the impact of the determinants of antibiotic prescriptions on the actual prescribing quality of each physician.

Ethics and dissemination The project has been approved by the ethics committee of the Regional Health Trust of Sardinia (176/2019/CE, 24 September 2019). The results will be useful to inform evidence-based interventions to tackle irrational antibiotic use in the community.

\section{INTRODUCTION}

Antimicrobial resistance (AMR) has emerged, alongside climate change, as a global threat to public health and wealth. ${ }^{1}$ Every year in Europe 33000 people die due to infections

\section{Strengths and limitations of this study}

- The use of internationally accepted indicators of antibiotic consumption developed by European Surveillance of antibiotic Consumption (ESAC)-net and the comprehensiveness of the data source selected to perform this study allows comparability to similar studies performed across Europe and enhances external validity of the results.

- The questionnaire that will be used to assess factors deemed to influence antibiotic prescribing practices of general practitioners is the cross-cultural adaptation of a fully validated scale in Spanish language and the adapted version will undergo a full validation study to maintain the psychometric properties of the original version of the scale.

- The main limitation of the study is that no clinical data about the reason for the encounter resulting in an antibiotic prescription is available.

- This limitation is addressed by the use, on the weighted population of each physician, of the ESAC quality indicators, specifically developed and validated to be used on administrative databases; furthermore, the use of this indicators will avoid bias resulting from inaccurate registration of the reason for the encounter.

caused by resistant bacteria and, globally approximately 700000 cases of such infections occur every year. ${ }^{23}$ According to current estimates, AMR is causing an excess of healthcare system costs up to US $\$ 1$ billion and a loss of gross domestic product (GDP) of US $\$ 3$ trillion per year. ${ }^{4}$ By 2050, antimicrobial resistant bacteria will cause more than 10 million deaths each year and a global loss of GDP of more than US $\$ 100$ trillion. $^{5}$

The overuse of antibiotics is one of the main drivers of AMR, but on a global scale antibiotic use is still rising. ${ }^{6-9}$ Italy has a higher 
consumption rate of antibiotics compared with many other European countries. ${ }^{10}$ In 2018, the annual consumption rate of Italian hospital and primary care sectors combined was 21.4 defined daily doses (DDDs)/1000 inhabitants/ day (DIDs) and the vast majority of antibiotic prescriptions (ie, 90\%, 19 DIDs) are filled by general practitioners (GPs). Aggregated level data show that there is a high variability of antibiotic prescription rates among different regions of the country and a high variability of antibiotic prescription rates in different seasons of the year, with highest rates occurring in influenza season. ${ }^{11}$ The finding of a strong seasonal variability seems to be consistent with previous studies that showed that, in general practice, the majority of antibiotics are prescribed for acute respiratory infections, such as acute bronchitis, otitis media, sinusitis or pharyngitis. In these conditions, according to guidelines, antibiotics are not routinely recommended and often inappropriately prescribed. ${ }^{12} 13$

Moreover, the consumption of broad-spectrum antibiotics is approximately twice as high as in other European countries like Norway, Sweden or the UK. ${ }^{10}$

For all these reasons it seems to be very likely that the majority of antibiotic prescriptions in Italy could be avoided. Both, qualitative and quantitative methodologies have been used in different settings of low-income, middle-income and high-income countries to understand the determinants of antibiotic overuse. ${ }^{14-19}$ Prescribing, especially antibiotic prescribing, is a complex behaviour, with intrinsic factors (sociodemographic variables, knowledge, attitudes) and extrinsic factors (signs and symptoms at presentation, comorbidity, sociodemographic variables, workload, influence of pharmaceutical companies, cost saving) that impact the healthcare professional decision. ${ }^{20}$ Therefore, to inform stakeholders (GPs, European and national agencies, scientific societies) in the development of effective interventions to tackle irrational antibiotic use, an understanding of the context specific determinants of antibiotic prescription is needed. ${ }^{20} 21$

GPs have a key role in Italy's National Health System (NHS). Virtually all of the resident population is compulsorily registered on a GP list and a pharmaceutical prescription can be charged to the NHS only if filled by a GP or a specialist consultant working directly for the NHS. Consequently, taking into consideration that in public healthcare systems GPs act as pharmaceutical gatekeepers, they could play an active role in tackling the phenomenon of antibiotic overuse and non-evidencebased prescription habits. ${ }^{22}$

At the best of our knowledge, there are no published studies that measure, with validated tools, the knowledge and attitudes of GPs on AMR and antibiotic prescriptions in Italy. Moreover, we were not able to retrieve any study that evaluated the influence of knowledge and attitudes of GPs on their prescriptions of antibiotics in our settings.

Findings of this study will be useful to develop evidencebased physician-targeted interventions to improve the quality of antibiotic prescriptions among Italian GPs. Moreover, this study could serve as a generalisable methodological framework to gain an understanding of GPs prescription practices and, therefore, to develop effective interventions to foster good clinical practice in primary care.

\section{AIMS AND OBJECTIVES}

The study will be divided into two distinct but related parts: study axis A and study axis B.

\section{Study axis A}

Aim

To describe the attitudes and knowledge of GPs regarding AMR and antibiotic prescriptions by administering a questionnaire adapted from Spanish to Italian language, namely the Knowledge and Attitudes on Antibiotics and Resistance questionnaire (KAAR-11) for primary care physicians, adapted to Italian language and culture (ITA-KAAR). ${ }^{23}$

\section{Objectives}

1. Measure the attitudes and knowledge of GPs through the administration of the ITA-KAAR questionnaire.

2. Describe the characteristics and working context of GPs by filling a sociodemographic form.

3. Evaluate the reliability and validity of the ITA-KAAR questionnaire.

\section{Study axis B}

Aim

To describe the quantity, pharmacological classes, appropriateness and interindividual variability of GPs prescriptions of antibiotics in the Italian region of Sardinia and to investigate the determinants of inappropriate prescriptions.

\section{Objectives}

1. Evaluate GPs antibiotic prescribing behaviour (quantity, pharmacological class, appropriateness, interindividual variability) through the analysis of prescription data of antibiotics for systemic use recorded in the administrative database of the Regional Health Trust of Sardinia (Azienda Tutela Salute, Sardegna).

2. Evaluate the association between the determinants of GPs prescribing behaviour (demographic factors, work environment, territorial context, attitudes and knowledge regarding antibiotics and resistance, resulting from the objectives A1 and A2) and the appropriateness of antibiotic prescriptions (resulting from the objective $\mathrm{B} 1$ ).

\section{METHODS AND ANALYSIS Study design}

A cross-sectional study will be carried out for both study axis A and study axis B. 


\section{Study axis $A$}

An attitudes and knowledge questionnaire (ITA-KAAR) and a sociodemographic form will be administered to all GPs working in the Italian region of Sardinia, to measure attitudes, knowledge and sociodemographic and workcontext related factors deemed to be associated with AMR and antibiotic prescriptions.

\section{Study axis B}

A drug utilisation study will be conducted to evaluate the prescriptions and the appropriateness of antibiotic choice for systemic use of all Sardinian GPs and to investigate the influence of determinants of inappropriate prescription.

\section{Study population and setting}

All residents of the Sardinia Region are covered by the NHS, which provides comprehensive hospital and outpatient care for those registered with a GP. The GPs provide primary care for the whole temporarily or permanently resident population and a pharmaceutical prescription must be filled by a GP or a specialist consultant to be charged to the NHS. $^{24}$

\section{Study axis $A$}

The entire Sardinian GP workforce $(n=1200)$, providing care to 1639591 Sardinian inhabitants at the date of 1 January 2019, will be recruited. ${ }^{25}$ The ITA-KAAR questionnaire and a sociodemographic form will be filled by all GPs included in the study through an online platform.

\section{Study axis B}

All prescriptions of antibiotics for systemic use (Anatomical Therapeutic Classification-ATC J01) filled by Sardinian GPs will be extracted from the pharmaceutical database of the Regional Health Trust of Sardinia during the period 2017-2019. The database contains all prescriptions covered by the NHS and is regularly updated.

\section{Outcome and covariates assessment}

\section{Study axis A}

The knowledge and attitudes section of ITA-KAAR measures, by means of a Visual Analogue Scale (VAS), some of the GPs attitudes on AMR and antibiotic prescriptions, namely lack of knowledge, fear, external responsibility and complaisance to patient's demands and expectations. The 7-item accessorial section of the questionnaire focuses on some of the main information sources for good clinical practice deemed to be relevant for GPs.

The questionnaire, including an additional section assessing sociodemographic and work-context related factors potentially associated with the appropriateness of antibiotic use, will be sent through an online platform via email to all GPs of the region of Sardinia. Reminders will be sent to non-responders every 10-14 days. The first 100 responders will be asked to complete the questionnaire a second time after 10-14 days (test-retest reliability). After this first phase of data collection, in case of low response rate, a comprehensive stepwise approach (including
Box 1 ITA-KAAR questionnaire, main section exploring physician knowledge and attitudes

ITA-KAAR questionnaire

Knowledge and attitudes regarding antibiotics and resistance

1. Antibiotic resistance is a major public health problem in our setting.

2. In primary care it is useful to wait for microbiology results before treating infectious diseases.

3. Prescribing an antibiotic to a patient does not influence the development of resistance.

4. New antibiotics will be developed to solve the problem of resistance.

5. The use of antibiotics in animals is a major cause of the occurrence of new resistance.

6. When in doubt, it is better to ensure that a patient is cured of an infection by using a broad-spectrum antibiotic.

7. I often prescribe antibiotics because it is impossible to track the patient accurately.

8. When in doubt as to whether a patient has a bacterial disease, it is best to prescribe an antibiotic.

9. I often prescribe antibiotics because patients ask me for them.

10. If a patient believes that he needs an antibiotic and the doctor does not prescribe it, he will get it at the pharmacy without a prescription.

11. Amoxicillin is useful for resolving most respiratory infections in primary care.

phone calls, postal reminders, reminders at continuous medical education events, involvement of locally known network leaders, professional associations and the state medical boards) will be used to achieve an acceptable response rate (see the Sample size section). ${ }^{26}$

The KAAR-11 questionnaire has shown to be a reliable and valid instrument to assess attitudes and knowledge of GPs on AMR and antibiotic prescriptions. The questionnaire was developed taking into account the findings of a literature review and a qualitative focus group study. ${ }^{20} 27$

The ITA-KAAR (KAAR-11 adapted to Italian language and culture, box 1) has been produced using the crosscultural adaptation methodology described by Beaton and colleagues. $^{28}$

Four professional translators and two bilingual physicians performed independently three forward and three backward translations of the original Spanish questionnaire. All the translators were naïve to the aims and scopes of the study. An expert committee, composed by primary care physicians, research methodologists, a mental health expert, a university professor for pharmacology, a university professor for microbiology, two university professors for Italian and Spanish language and all the involved translators, reviewed the translations and produced a prefinal version of the questionnaire. A pilot study was carried out by administering the prefinal version of ITA-KAAR to an convenience sample of the target population (45 GPs working in different regions of Italy). Among these, two GPs from the Sardinian region participated in the pilot study of the cross-cultural adaptation process and will be therefore excluded from the study (axis A). Comments and suggestions of the responders were registered and the necessary amendments to the prefinal version were 
made to produce the final version of the ITA-KAAR. The detailed process of cross-cultural adaptation and preliminary validation are described elsewhere. ${ }^{29}$

An English version of ITA-KAAR and the sociodemographic form is provided in online supplementary file 1 .

\section{Study axis B}

The Regional Health Trust of Sardinia will provide the data of all prescriptions of antibiotics for systemic use (ATC J01) filled by GPs between 1 January 2017 and 31 December 2019. For each GP and for each year of the study, DDDs and DIDs of the prescribed antibiotics and European Surveillance of antibiotic Consumption (ESAC) quality indicators for outpatient antibiotic use ${ }^{30}$ will be calculated.

The following ESAC quality indicators will be considered:

1. J01_DID: consumption of antibacterials for systemic use (ATC J01) expressed in DID.

2. J01C_DID: consumption of penicillins (ATC J01C) expressed in DID.

3. J01D_DID: consumption of cephalosporins (ATC J01D) expressed in DID.

4. J01F_DID: consumption of macrolides, lincosamides and streptogramins (ATC J01F) expressed in DID.

5. J01M_DID: consumption of quinolones (ATC J01M) expressed in DID.

6. J01CE_\%: consumption of b-lactamase-sensitive penicillins (ATC J01CE) expressed as a percentage.

7. J01CR_\%: consumption of combinations of penicillins, including b-lactamase inhibitors (ATC J01CR), expressed as a percentage.

8. J01DD+DE_\%: consumption of third and fourth generation cephalosporins (ATC J01DD+J01 DE) expressed as a percentage.

9. J01MA_\%: consumption of fluoroquinolones (ATC J01MA) expressed as a percentage.

10. J01_B/N: ratio of the consumption of broadspectrum (ATC J01CR+J01DC+J01DD+J01F excluded J01FA01) to the consumption of narrow spectrum penicillins, cephalosporins and macrolides (ATC J01CE+J01DB+J01FA01).

11. J01_SV: seasonal variation in total antibiotic consumption (ATC J01).

12. J01M_SV: seasonal variation in quinolone consumption (ATC J01M).

To take into account the different prevalence of consumption of antibiotics by patient's age and gender, all the indicators will be calculated on the weighted population (based on the weights set up by the Department of Programming of the Italian Ministry of Health, online supplementary file 2) of the patients registered in the list of each physician. In particular, patients will be classified in age and gender classes; the number of subjects in each age and gender class will be multiplied by the corresponding weight; the sum of the values obtained will be proportionally reported to the regional population.
For each physician, a better performance in half plus one of the ESAC quality indicators compared with the median of the region, will be considered as an indicator of adequate quality of prescriptions of antibiotics (AQPA).

In order to better describe the variability of antibiotic prescribing behaviour of the study subjects in terms of use of first and second line drugs, for each GP and for each year of the study, prescriptions of antibiotics for systemic use (ATC J01) will be classified in the three groups of the WHO Essential Medicines List Access, Watch, and Reserve (AWaRe) classification. ${ }^{31}$

For the correspondence between ATC and WHO Essential Medicines List AWaRe classification see the online supplementary file 3 .

\section{Statistical analysis}

All statistical analysis will be performed using $\mathrm{R}$ or MPLUS. $^{32}{ }^{33}$ Continuous variables will be described by mean and SD and, in case of asymmetric distributions, the median and IQR will be used. Categorical variables will be represented through frequency distributions (absolute and percentage values). Comparisons between groups will be made, for continuous variables through the $\mathrm{t}$ test (Mann-Whitney $\mathrm{U}$ test where necessary), while for categorical variables through the $\chi^{2}$ test (Fisher's exact test where necessary). Statistical significance will be expressed in terms of $p$ values $(p \leq 0.05)$ and the $95 \%$ CIs will be added to the point estimates.

\section{Study axis A}

Handling of missing data will be dealt by imputation methods if the percentage of missing values is low $(<3 \%$ for each item). Otherwise, observations with missing values will be excluded from the analysis.

Cronbach's alpha will be used to assess the internal consistency of the total scale. The correlations between the items of the questionnaire will also be examined: items with a correlation lower than 0.30 will be considered for removal from the questionnaire, as well as items whose exclusion leads to an improvement in Cronbach's alpha value of 0.001 or more. To evaluate the test-retest reliability, the Pearson correlation coefficients will be calculated between the scores at the first and second administration. Furthermore, the intraclass correlation coefficients will be calculated.

The factorial structure of the ITA-KAAR will be evaluated through an exploratory factor analysis (EFA), using the maximum likelihood estimation (MLE). The number of factors to be included in the EFA will be decided after (1) evaluation of the number of eigenvalues $\geq 1$, (2) the screenplot and (3) the parallel analysis. The following goodness of fit indexes will be used to evaluate the different models: Tucker-Lewis Index $\geq 0.95$ and $\geq 0.90$ indicating a good or sufficient fit, respectively and the root mean square error of approximation $\leq 0.05$ or $\leq 0.08$ indicating good or sufficient fit, respectively. The correlation between factors will be estimated using the Pearson coefficient. Indicators with loadings less than 0.30 will be 
considered for the removal from the questionnaire. Path diagrams will be used to compare the loadings of each factor.

To evaluate the known-group validity, the medians of the questionnaire as a whole, for groups of items that explore the same domains and for the single items of the ITA-KAAR for the two groups of opposing doctors will be compared. These groups will be identified as the first and last decile of the percentage of ESAC indicators that, for each doctor, are greater than the reference value (median of the region).

To determine the ability of the ITA-KAAR in discriminating the prescribers with $(\mathrm{AQPA}=1)$ from those with inadequate quality of prescriptions $(\mathrm{AQPA}=0)$, a Receiver operatin characteristic (ROC) analysis will be performed with evaluation of the area under the curve (AUC). The following scheme will be used for the interpretation of the $\mathrm{AUC}$ values: $\mathrm{AUC}=0.5$ : no discriminative ability; $0.5<\mathrm{AUC}<0.7$ : inaccurate discriminative ability; $0.7<$ AUC $<0.9$ : moderately accurate discriminative ability; $0.9<$ AUC $<1.0$ : highly accurate discriminative ability; $\mathrm{AUC}=1.0$ : perfect discriminative ability.

\section{Study axis B}

The analysis of the association between ESAC quality indicators (dependent variable), sociodemographic factors and the answers to the ITA-KAAR questionnaire (explanatory variables) will be conducted through a Poisson regression model with a robust estimator of variance and will be expressed in terms of prevalence risk ratio. ${ }^{34}$

Each GP will be classified according to the AQPA, converted in a dichotomous dependent variable: $A Q P A=1$ (adequate quality of prescriptions) versus $\mathrm{AQPA}=0$ (inadequate quality of prescriptions). The explanatory variables of the model will be represented by the previously validated set of items of the questionnaire, together with the sociodemographic characteristics of the GPs, as follows: age and gender of the prescriber, number of registered patients, year of graduation, degree of urbanisation of the area where the doctor practises, GP training, specialisation, availability of a receptionist/back office, average number of daily consultations, access by appointment/ free access, average minutes per consultation.

The variables associated with the outcome in the univariate analysis $(p<0.10)$ will be considered eligible for inclusion in the multivariate model. The final model will be obtained through a stepwise forward selection method with a stopping rule based on the Akaike information criterion. ${ }^{35}$

\section{Sample size}

The calculation of the sample size (number of GPs to be interviewed) was performed on the basis of the results of a previous study ${ }^{36}$ and a literature review. ${ }^{37}$ Assuming a log-normal distribution, a difference in the medians of each item between the compared groups $(\mathrm{AQPA}=1 \mathrm{vs}$ $\mathrm{AQPA}=0)$ of 0.25 points on the VAS and an SD of 0.83 (corresponding to a range of variability of $99 \%$ of the values per single item equal to half of the VAS), a population of 1200 GPs, a response rate equal to or greater than $40 \%(n=480)$ guarantees a power of the association study greater than $80 \%{ }^{38}$

\section{Patient and public involvement}

GPs developed the research questions, the overall conception of the project and the present research protocol in collaboration with epidemiologists and public health professionals, in order to gain a better understanding of their own prescribing behaviours. Local medical boards are involved in the dissemination of the ITA-KAAR questionnaire and in a peer-led dissemination of the results of this study.

\section{ETHICS AND DISSEMINATION}

This project has been approved by the Ethics committee of the Regional Health Trust of Sardinia (protocol number 176/2019/CE, 24 September 2019).

The results of this study will be disseminated through publications in peer reviewed journals, relevant meetings, congresses and teaching sessions addressed to GPs and the public.

\section{DISCUSSION}

There are few doubts that AMR is strongly related to antibiotic consumption. ${ }^{6}$ Prescribing is a complex process and, as highlighted by Teixeira in a systematic review of qualitative studies, antibiotic prescribing is influenced by multiple determinants. ${ }^{20}$

Our study is intended to uncover the relationship between some of the most important hypothesised determinants of antibiotic prescriptions and the quality of prescription itself by using a theoretical framework of antibiotic prescribing behaviour. ${ }^{20}$ This model considers both, intrinsic and external factors to the healthcare professional that influences antibiotic prescription habits of primary care physicians (figure 1).

Despite the comprehensiveness of the proposed theoretical model, few studies tested it in practice. ${ }^{36} 39$ Therefore, we have designed a study to fill this lack of knowledge using the widely accepted knowledge, attitudes and practice survey methodology to assess the educational needs of GPs on AMR and antibiotic prescriptions.

The chosen study design has a number of strengths.

First, the ITA-KAAR, Italian version of the KAAR-11 questionnaire, which is the only fully validated scale developed to assess knowledge and attitudes of GPs regarding AMR and antibiotic prescriptions, was translated and adapted to Italian using a methodology aimed to maintain the psychometric properties of the original version of the scale s. $^{29}$ and will undergo a full validation study.

Second, in order to fully validate the ITA-KAAR against an external 'gold standard' (known-group validity) and to measure the magnitude of the association between each explanatory variable and the quality of antibiotic 


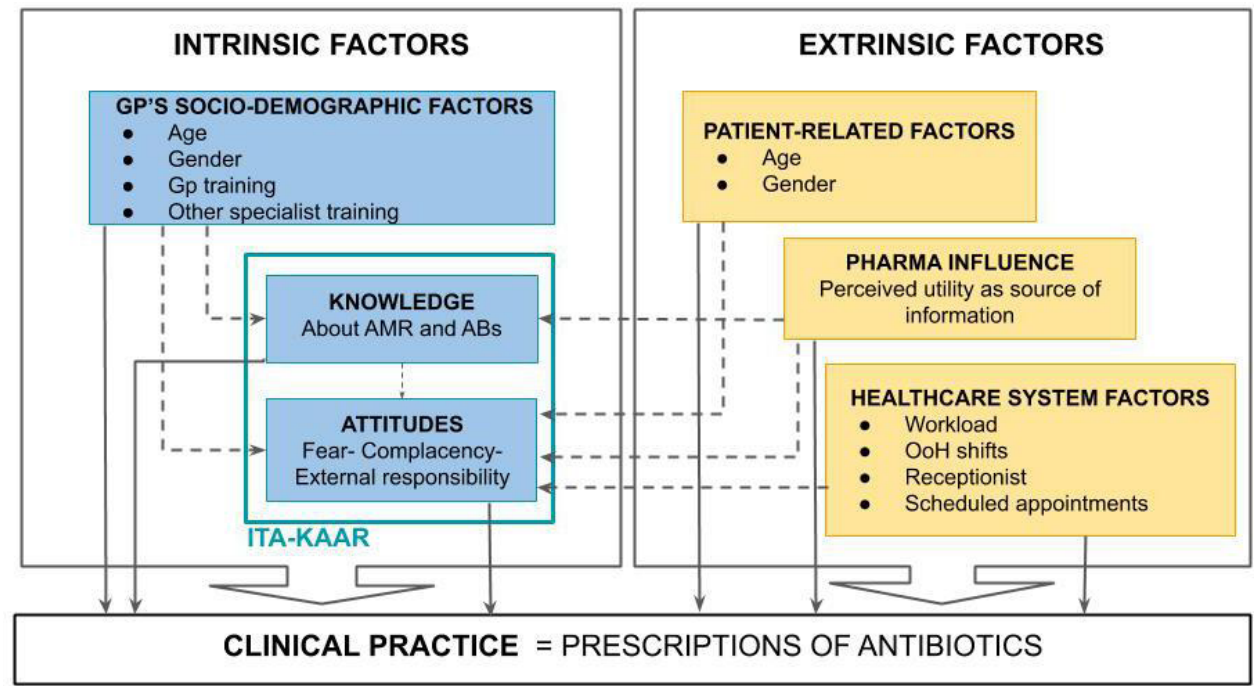

Figure 1 Theoretical model of antibiotic prescribing behaviour of GPs, adapted from Teixeira Rodrigues et al. ${ }^{20}$ Continuous arrows represent factors that are deemed to influence antibiotic prescriptions directly. Dotted arrows represent factors that are deemed do influence antibiotic prescriptions indirectly through the influence of knowledge and/or attitudes. ABs, antibiotics; AMR, antimicrobial resistance; GP, general practitioner; ITA-KAAR, Knowledge and Attitudes on Antibiotics and Resistance Italian version; $\mathrm{OoH}$, out of hours; Pharma, pharmaceutical company.

prescription, we will use a set of valid, reliable and internationally accepted indicators, ${ }^{30}$ calculated using data from clinical practice. This leads to further strengths of our study design: (1) they allow comparability to similar studies performed across Europe; (2) their use excludes any opportunistic selection of indicators for the purpose to meet the study objectives.

Third, the categorisation of the prescriptions of antibiotics for systemic use (ATC J01 subclasses) according to the WHO AWaRe classification, will enhance the comparability of the results of the present study to these of similar studies performed globally ${ }^{4041}$ and will be useful to shape an appropriate communication strategy in the context of antibiotic stewardship interventions targeted to GPs in the Sardinia region and nationally. ${ }^{42}$

Fourth, the data source of the drug utilisation study (study axis B) is an administrative database containing any antibiotic prescription covered by the NHS, filled by every single GP in the examined period of time. This completeness is a well-known strength of drug utilisation studies performed on large administrative databases, that have been widely used as reliable data sources among European countries to inform policy. ${ }^{434}$

A further strength of the study refers to the estimation methodology that will be used to measure the magnitude of the association between the hypothesised determinants of antibiotic prescriptions and the real prescription data used in this study, namely a multivariate Poisson model with robust estimation of variance. This model is generally preferred to the traditional logistic model in crosssectional studies when the dependent variable is not rare. In fact, in the case of frequent outcomes, typical in crosssectional studies, the OR, obtained from the application of the traditional logistic model, can lead to a strong overestimation of the prevalence ratio and therefore of the magnitude of the association. Moreover, similarly to the multivariate logistic model, the Poisson model produces association measures that are adjusted with respect to the other confounding factors. ${ }^{34}$

Some potential limitations need to be mentioned.

First, the response rate to a questionnaire could be low even if a comprehensive data collection strategy is planned. ${ }^{45}$ To minimise non-response bias (a type of selection bias) and exclude the risk that responders differ in meaningful ways from non-responders, a subgroup analysis of responders and non-responders to the questionnaire will be performed in relation to each ESAC indicator. In particular, for both subgroups the percentages of GPs who have shown an $\mathrm{AQPA}=1$ or an $\mathrm{AQPA}=0$ as defined above, will be calculated. In case of differences that do not reach statistical significance between the two subgroups, non-response bias could be reasonably excluded.

A second limitation could be that the ESAC quality indicators ${ }^{30}$ used in our study are not developed to consider the underlying reason of the prescription (ie, the diagnosis). The choice of these indicators above others that take into account the clinical reason for encounter ${ }^{46}$ was made because data regarding the underlying diagnosis are not available in our setting (GP health records that could be used for research purpose does not exist in the region of Sardinia). Nevertheless, we feel that the ESAC quality indicators without the underlying clinical diagnosis ${ }^{30}$ are appropriate indicators for the purposes of our study. In fact, after adjustment for patients age and gender, we have no reason to believe that the population of patients registered in the list of any single GP differ from each other significantly with respect of the occurrence neither of diseases for which antibiotics are currently prescribed in general practice, nor for other conditions that could act as relevant confounding factors. 


\section{Future perspectives}

Considering that $90 \%$ of antibiotic prescriptions in human health are filled by GPs, the results of this study will be useful to inform stakeholders on the educational needs and the context specific determinants of antibiotic use and will serve the development and implementation of further targeted interventions to reduce antibiotic consumption and prescriptions in the community, namely ${ }^{47}$ :

1. The design, administration and implementation of a multifaceted intervention targeted to the needs of physicians, as revealed in the first phase of the study.

2. The assessment, in a prospective design, of the impact of this intervention on reducing antibiotic prescriptions.

Due to the fact that determinants of inappropriate use of antibiotics are context-specific, the overall aim of the 'Broad Spectrum' project is to create a methodological framework to be applied in other Italian and European regions to tackle inappropriate antibiotic use, thereby reducing and preventing the spread of antibiotic resistance.

\section{Author affiliations}

${ }^{1}$ Department of Medical Science and Public Health, University of Cagliari, Cagliari, Italy

${ }^{2}$ General Practitioner in Training, Primary Care Department, Regional Health Trust of Sardinia, Cagliari, Italy

${ }^{3}$ General Practitioner in Training, Primary Care Department, Local Health Trust Modena, Modena, Italy

${ }^{4}$ National Centre for Pre-Clinical and Clinical Drug Research and Surveillance (CNRVF), Istituto Superiore di Sanita, Rome, Italy

${ }^{5}$ Department of Preventive Medicine and Public Health, Universidade de Santiago de Compostela, Santiago de Compostela, Spain

${ }^{6}$ General Practitioner, Primary Care Department, Regional Health Trust, Cagliari, Italy ${ }^{7}$ Mailman School of Public Health, Columbia University, New York, New York, USA

${ }^{8}$ Health Technology Assessment Unit, Regional Health Trust of Sardinia, Cagliari, Italy

${ }^{9}$ Brayford Square Surgery, Tower Hamlets Primary Care Trust, London, UK

Acknowledgements This project is funded by a grant from the Foundation of Sardinia and is conducted within a scientific collaboration between the Italian National Institute of Health and the Department of Medical Science and Public Health of the University of Cagliari (Resolution of Italian National Institute of Health, 17 December 2019). The authors thank Micaela Morelli, vice-president of the University of Cagliari, Emilio Montaldo (GP, specialist in allergology and immunology, member of the Central Committee of the Italian National Federation of Medical Boards) and Raimondo lbba (president of the Medical Board of the Province of Cagliari, Italy) for their substantial contribution in fostering the Broad Spectrum project at an institutional level.The authors would like to thank also Maria Teresa Zedda (GP, specialist in allergology and immunology), Orietta Massidda (professor for Clinical Microbiology, University of Trento, Italy) for their advices and encouragements and Noel Baxter (GP, Southwark, London, UK) for his critical revision of this work.

Contributors PKK, AS and MN developed the research question. PKK and AS designed, together with the substantial contribution of SSA, MarMas, RDC, MGC, VF, AF and MFM, the overall conception of the project. PKK wrote the protocol and, together with AS, LM and AP the project proposal. PKK and AS, in collaboration with SSA, MarMas, RDC, wrote the first draft of the manuscript. All authors read the manuscript, made relevant critiques and amendments and approved the final draft. PKK, AS, VF, FC, AP, LM, MauMar and MatMas will collect data. MarMas, SSA, RDC, MFM, MGC and PKK will perform data analysis.

Funding Public Health, Preventive Medicine and Rehabilitation Grant programme (Foundation of Sardinia, Grant n. 30718).

Disclaimer The views expressed are those of the authors and are not necessarily those of the Italian National Institute of Health, the University of Cagliari, the University of Santiago de Compostela, the Columbia University, the Local Health Trust of Modena or the Regional Health Trust of Sardinia.
Competing interests None declared.

Patient and public involvement Patients and/or the public were involved in the design, or conduct, or reporting, or dissemination plans of this research. Refer to the Methods section for further details.

Patient consent for publication Not required.

Ethics approval Ethics committee of the Regional Health Trust of Sardinia (protocol number 176/2019/CE, 24th September 2019)

Provenance and peer review Not commissioned; externally peer reviewed.

Open access This is an open access article distributed in accordance with the Creative Commons Attribution Non Commercial (CC BY-NC 4.0) license, which permits others to distribute, remix, adapt, build upon this work non-commercially, and license their derivative works on different terms, provided the original work is properly cited, appropriate credit is given, any changes made indicated, and the use is non-commercial. See: http://creativecommons.org/licenses/by-nc/4.0/.

ORCID iDs

Peter Konstantin Kurotschka http://orcid.org/0000-0003-3750-6147

Mauro Giovanni Carta http://orcid.org/0000-0003-0706-9687

\section{REFERENCES}

1 ECDC/EMEA Joint Technical Report. The bacterial challenge: time to react, 2009. Available: https://ecdc.europa.eu/en/publications-data/ ecdcemea-joint-technical-report-bacterial-challenge-time-react

2 European Commission. A European one health action plan against antimicrobial resistance (AMR), 2017. Available: https://ec.europa.eu/ health/amr/sites/amr/files/amr_action_plan_2017_en.pdf

3 Cassini A, Högberg LD, Plachouras D, et al. Attributable deaths and disability-adjusted life-years caused by infections with antibioticresistant bacteria in the EU and the European economic area in 2015: a population-level modelling analysis. Lancet Infect Dis 2019;19:56-66.

4 Naylor NR, Atun R, Zhu N, et al. Estimating the burden of antimicrobial resistance: a systematic literature review. Antimicrob Resist Infect Control 2018;7:58.

5 O'Neil J. Tackling drug-resistant infections globally: final report and recommendations. 1. United Kingdom, 2016.

6 Bell BG, Schellevis F, Stobberingh E, et al. A systematic review and meta-analysis of the effects of antibiotic consumption on antibiotic resistance. BMC Infect Dis 2014;14:13.

7 Shallcross LJ, Davies DSC. Antibiotic overuse: a key driver of antimicrobial resistance. Br J Gen Pract 2014;64:604-5.

8 Klein EY, Van Boeckel TP, Martinez EM, et al. Global increase and geographic convergence in antibiotic consumption between 2000 and 2015. Proc Natl Acad Sci U S A 2018;115:E3463-70.

9 Van Boeckel TP, Gandra S, Ashok A, et al. Global antibiotic consumption 2000 to 2010: an analysis of national pharmaceutical sales data. Lancet Infect Dis 2014;14:742-50.

10 European Centre for Disease Prevention and Control. Antimicrobial consumption surveillance in Europe 2018. annual report of the European antimicrobial consumption surveillance network (ESACNet. Stockholm: ECDC, 2019.

11 Osservatorio Nazionale sull'impiego dei Medicinali. L'uso degli an bio ci in Italia. Rapporto Nazionale 2018. Roma: Agenzia Italiana del Farmaco, 2019.

12 Fleming-Dutra KE, Hersh AL, Shapiro DJ, et al. Prevalence of inappropriate antibiotic prescriptions among US ambulatory care visits, 2010-2011. JAMA 2016;315:1864-73.

13 Havers FP, Hicks LA, Chung JR, et al. Outpatient antibiotic prescribing for acute respiratory infections during influenza seasons. JAMA Netw Open 2018;1:e180243.

14 Ciofi degli Atti ML, Massari M, Bella A, et al. Clinical, social and relational determinants of paediatric ambulatory drug prescriptions due to respiratory tract infections in Italy. Eur J Clin Pharmacol 2006;62:1055-64.

15 Harbarth S, Monnet DL. Cultural and socioeconomic determinants of antibiotic use. In: Antibiotic policies: fighting resistance, 2005: 29-40.

16 Masiero G, Filippini M, Ferech M, et al. Socioeconomic determinants of outpatient antibiotic use in Europe. Int $J$ Public Health 2010;55:469-78.

17 Touboul-Lundgren P, Jensen S, Drai J, et al. Identification of cultural determinants of antibiotic use cited in primary care in Europe: a mixed research synthesis study of integrated design "Culture is all around us". BMC Public Health 2015;15:908. 
18 Godman B, Haque M, McKimm J, et al. Ongoing strategies to improve the management of upper respiratory tract infections and reduce inappropriate antibiotic use particularly among lower and middle-income countries: findings and implications for the future. Curr Med Res Opin 2020;36:301-27.

19 Giusti A, Spila Alegiani S, Ciofi Degli Atti ML, et al. Surgical antibiotic prophylaxis in children: a mixed method study on healthcare professionals attitudes. BMC Pediatr 2016;16:203.

20 Teixeira Rodrigues A, Roque F, Falcão A, et al. Understanding physician antibiotic prescribing behaviour: a systematic review of qualitative studies. Int J Antimicrob Agents 2013;41:203-12.

21 Dyar OJ, Beović B, Vlahović-Palčevski V, et al. How can we improve antibiotic prescribing in primary care? Expert Rev Anti Infect Ther 2016;14:403-13.

22 Colliers $\mathrm{A}$, Coenen $\mathrm{S}$, Philips $\mathrm{H}$, et al. Optimising the quality of antibiotic prescribing in out-of-hours primary care in Belgium: a study protocol for an action research project. BMJ Open 2017;7:e017522.

23 López-Vázquez P, Vázquez-Lago JM, Gonzalez-Gonzalez C, et al. Development and validation of the knowledge and attitudes regarding antibiotics and resistance (KAAR-11) questionnaire for primary care physicians. J Antimicrob Chemother 2016;71:2972-9.

24 Accordo Collettivo Nazionale per la disciplina. Accordo Collettivo Nazionale per La disciplina dei rapporti Con I medici di medicina generale Al sensi dell'art 8 del D.Lgs. N. 502 del 1992 E successive modificazioni E integrazioni, 2018.

25 ISTAT. Direzione centrale per Le statistiche E Le indagini sulle istituzioni sociali, Popolazione residente al $1^{\circ}$ gennaio, 2019. Available: http://demo.istat.it

26 Ebert JF, Huibers L, Christensen B, et al. Paper- or web-based questionnaire invitations as a method for data collection: crosssectional comparative study of differences in response rate, completeness of data, and financial cost. J Med Internet Res 2018;20:e24.

27 Teixeira Rodrigues A, Ferreira M, Roque F, et al. Physicians' attitudes and knowledge concerning antibiotic prescription and resistance: questionnaire development and reliability. BMC Infect Dis 2016;16:7.

28 Beaton DE, Bombardier C, Guillemin F, et al. Guidelines for the process of cross-cultural adaptation of self-report measures. Spine (Phila Pa 1976) 2000;25:3186-91.

29 Kurotschka PK, Moro MF, Demurtas J, et al. [General practitioners knowledge and attitudes on antibiotics and resistance: cross-cultural adaptation and preliminary validation of KAAR-11 questionnaire.] Recenti Prog Med. In Press 2020;111:368-370.

30 Coenen S, Ferech M, Haaijer-Ruskamp FM, et al. European surveillance of antimicrobial consumption (ESAC): quality indicators for outpatient antibiotic use in Europe. Qual Saf Health Care 2007;16:440-5.

31 Sharland M, Pulcini C, Harbarth S, et al. Classifying antibiotics in the who essential medicines list for optimal use-be aware. Lancet Infect Dis 2018;18:18-20.

32 R Core Team (2019). R: a language and environment for statistical computing. R foundation for statistical computing V, Austria. Available: https://www.R-project.org/. Ao.
33 Muthén LK, Muthén BO. Mplus user's guide. Sixth Edn. Los Angeles C, 2007.

34 Barros AJD, Hirakata VN. Alternatives for logistic regression in crosssectional studies: an empirical comparison of models that directly estimate the prevalence ratio. BMC Med Res Methodol 2003;3:21.

35 Sakamoto Y, Kitagawa G, Masato I. Akaike information criterion statistics. New York: Springer, 1986.

36 Gonzalez-Gonzalez C, López-Vázquez P, Vázquez-Lago JM, et al. Effect of physicians' attitudes and knowledge on the quality of antibiotic prescription: a cohort study. PLoS One 2015;10:e0141820.

37 Anthoine E, Moret L, Regnault A, et al. Sample size used to validate a scale: a review of publications on newly-developed patient reported outcomes measures. Health Qual Life Outcomes 2014;12:176.

38 O'Keeffe AG, Ambler G, Barber JA. Sample size calculations based on a difference in medians for positively skewed outcomes in health care studies. BMC Med Res Methodol 2017;17:157.

39 Liu C, Liu C, Wang D, et al. Intrinsic and external determinants of antibiotic prescribing: a multi-level path analysis of primary care prescriptions in Hubei. China, 2019.

40 Robertson J, Iwamoto K, Hoxha I, et al. Antimicrobial medicines consumption in Eastern Europe and Central Asia - an updated crossnational study and assessment of quantitative metrics for policy action. Front Pharmacol 2018;9:1156.

41 Hsia Y, Lee BR, Versporten A, et al. Use of the who access, watch, and reserve classification to define patterns of hospital antibiotic use (aware): an analysis of paediatric survey data from 56 countries. Lancet Glob Health 2019;7:861-71.

42 Sharland M, Gandra S, Huttner B, et al. Encouraging awareness and discouraging inappropriate antibiotic use-the new 2019 essential medicines list becomes a global antibiotic stewardship tool. Lancet Infect Dis 2019;19:1278-80.

43 Latry P, Molimard M, Bégaud B, et al. How reimbursement databases can be used to support drug utilisation studies: example using the main french national health insurance system database. Eur J Clin Pharmacol 2010;66:743-8

44 Leporowski A, Godman B, Kurdi A, et al. Ongoing activities to optimize the quality and efficiency of lipid-lowering agents in the Scottish national health service: influence and implications. Expert Rev Pharmacoecon Outcomes Res 2018;18:655-66.

45 Aitken $\mathrm{C}$, Power R, Dwyer R. A very low response rate in an on-line survey of medical practitioners. Aust $N Z J$ Public Health 2008;32:288-9.

46 Adriaenssens N, Coenen S, Tonkin-Crine S, et al. European surveillance of antimicrobial consumption (ESAC): disease-specific quality indicators for outpatient antibiotic prescribing. BMJ Qual Saf 2011;20:764-72.

47 Teixeira Rodrigues A, Roque F, Piñeiro-Lamas M, et al. Effectiveness of an intervention to improve antibiotic-prescribing behaviour in primary care: a controlled, interrupted time-series study. J Antimicrob Chemother 2019;74:2788-96. 